\title{
Gut mechanosensors: enterochromaffin cells feel the force via PIEZO2
}

The gastrointestinal tract responds to luminal mechanical stimuli with fluid secretion and gastrointestinal motility, but the epithelial mechanosensors involved in this process were previously unclear. Now, a subset of enterochromaffin cells (ECs) are shown to be mechanosensitive, using PIEZO2 channels to couple force to serotonin release and intestinal secretion.

Studies in the late 1950s showed that mechanical stimulation of the gastrointestinal mucosa results in serotonin release and changes in gastrointestinal motility. At that time, serotonin-containing ECs the largest population of epithelial enteroendocrine cells (EECs) were proposed to be specialized epithelial mechanosensors that converted mechanical forces into serotonin release. However, whether

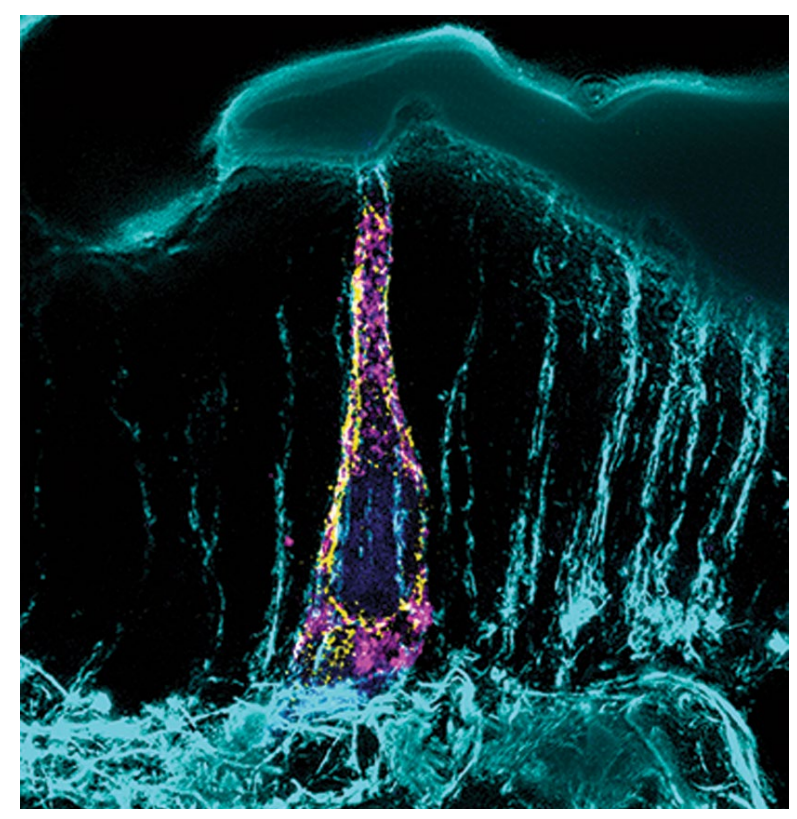

Super-resolution microscopy image of mouse jejunum fluorescently labelled for F-actin (cyan), PIEZO2 (yellow), serotonin (magenta) and TPH1 (blue). Image courtesy of A. Beyder. primary ECs were mechanosensitive was unresolved, leading to debate regarding their role in gastrointestinal mechanobiology. "Part of the uncertainty is because the mechanisms of mechanical release of serotonin - or even if enterochromaffin cells are mechanosensitive - was not answered," reports lead author Arthur Beyder. "We wanted to understand how EC cells work."

To test the hypothesis that ECs are mechanosensitive, the investigators first had to overcome the challenges that prevented progress in this field. "Epithelial cells don't survive well in primary cultures and ECs comprise $\sim 1 \%$ of epithelium, so they are difficult to identify in culture," explains Beyder. The researchers used cell lineage tracing techniques and mouse models to selectively label EECs transgenically and also developed primary culture methods to enable in vitro experimentation. They found that a subset of ECs express PIEZO2, a mechanosensitive ion channel also found in Merkel cells - the epithelial touch-sensitive skin cells. Using super-resolution microscopy, these channels were shown to be distributed near serotonin vesicles, suggesting functional coupling.

"The next challenge was that EECs are some of the smallest in your body (size of sperm), but we wanted to use patch clamps to directly test their mechanosensitivity. So, we developed a way to do electrical and mechanical recordings on EECs at the same time," says Beyder. These experiments showed that mechanical stimulation of a subset of isolated EECs led to a rapid inward current that was diminished when PIEZO2 was depleted or when PIEZO2

inhibitors were included.

To examine whether calcium signalling was downstream of PIEZO2 activation, primary cultured EECs were exposed to shear force. Stimulated cells responded with a prolonged increase in intracellular calcium, which was inhibited by PIEZO2 depletion. The investigators also stimulated planarized intestinal organoids with shear forces and found similar PIEZO2-dependent increases in intracellular calcium.

"We next wanted to test for serotonin release, but it is very hard to record from single cells, so we used a biosensor approach," says Beyder. In the majority of mechanosensitive EECs, mechanical stimulation evoked increase in intracellular calcium and serotonin release, which implicated ECs as the mechanosensitive enteroendocrine cells. After depleting PIEZO2, responses to force and from the serotonin biosensor were reduced, suggesting that the serotonin response to mechanical stimulation was dependent on PIEZO2. Finally, in conditional gut epithelium PIEZO2 knockout mice, pressure failed to produce a typical increase in fluid secretion, indicating an important physiological function for PIEZO2.

"We now need to understand the signalling pathways involved in mechanotransduction downstream of PIEZO2, whether other types of EECs are mechanosensitive, and if so, whether they use PIEZO2," concludes Beyder. "Our work may allow us to separate the physiological responses to chemical and mechanical luminal stimuli, which might enable targeted modulation of gastrointestinal physiology and potentially novel diagnostic and therapeutic opportunities in functional gastrointestinal diseases."

Iain Dickson

ORIGINAL ARTICLE Alcaino, C. et al. A population of gut epithelial enterochromaffin cells is mechanosensitive and requires Piezo2 to convert force into serotonin release. Proc. Natl Acad. Sci.USA 115, E7632-E7641 (2018) 